\title{
Analysis of Maximum Ratio Transmission in Ad Hoc Networks with Imperfect Channel State Information
}

\author{
Mingzhu Deng \\ College of Computer \\ National University of Defense Technology \\ Changsha, China \\ Email: dengmz@nscc-tj.gov.cn,
}

\author{
Guangming Liu \\ College of Computer \\ National University of Defense Technology \\ Changsha, China \\ Email:1gmnscc@yeah.net
}

\author{
Yuran $\mathrm{Hu}$ \\ College of Computer Science \\ Sichuan Normal University \\ Chengdu, China \\ Email: hyr9057@gmail.com
}

\begin{abstract}
In this paper, the performance of maximum ratio transmission (MRT) with imperfect channel state information (CSI) is analyzed. Different from previous works, a multiuser scenario is considered. By modeling the source nodes as a homogeneous Poisson point process, the outage probability is derived in closed form. Our results indicate the quality of CSI has great impact on the performance of MRT and imperfect CSI will cause certain diversity loss. Thus attention should be made on the design of CSI feedback.
\end{abstract}

Keywords-maximum ratio transmission (MRT), channel state information (CSI), ad hoc network, poisson point process

\section{INTRODUCTION}

Diversity techniques have been adopted in various systems to combat fading [1]. In a diversity system, signals received from different diversity branches are combined to achieve a better detection performance. In this paper, we mainly concern with the transmit diversity techniques. Generally, there are mainly three transmit diversity techniques: maximum ratio transmission (MRT), space time coding and transmit antenna selection. Compared with its counterparts, MRT can achieve not only full diversity but also array gain. Thus this paper will focus on MRT.

When MRT is adopted, the channel state information (CSI) is needed. However, perfect CSI is often not available due to channel estimation error and feedback delay. Thus the bit-error-rate (BER) of the conventional MISO system is analyzed in the presence of CSI feedback delay and estimation error in $[2,3]$. Unfortunately, these works only focus on the performance of a specific link and thus is not applicable to a multiuser scenario, such as ad hoc networks. In multiuser systems, the number of users and the users' locations are often dynamic, which cause certain difficulty in system modeling. Recently, Poisson point processes have been proposed as a reasonable model for multiuser network modeling, and its accuracy has been verified by various works $[4,5,6]$. Thus Poisson point process will be adopted to model the multiuser networks.

In this paper, the performance of MRT with imperfect CSI in an ad hoc network is analyzed. Here the imperfection of CSI comes from the delay of the CSI feedback. By modeling the nodes in the network as a homogeneous Poisson point process, the closed form expression of outage probability is derived. With the analytical results, the impact of imperfect CSI on the system performance is investigated and the result has shown the quality of CSI has a great impact on system performance. Thus certain attention is suggested to be made on the design of the CSI feedback. Furthermore, our result indicates only the diversity of 1 can be achieved in the presence of feedback error, i.e., imperfect CSI will cause certain diversity loss. According to our results, interference plays a non-negligible role and interference cancellation techniques can further improve the system performance.

The rest of this paper is organized as follows. System model is introduced in Section II. The outage performance of MRT is derived in Section III. Finally, conclusions are drawn in Section IV.

\section{SYSTEM MODEL}

An ad hoc network is considered here. The source nodes are modeled by a homogeneous Poisson point process $\Phi$ with intensity $\lambda$. Receivers are located in a given fixed distance D from the corresponding source nodes in a random orientation. Transmitters are equipped with $N$ antennas and receivers have only single antenna. Each source node transmits to their associated receiver by maximum ratio transmission (MRT) with power $\mathrm{P}$. The homogeneity of $\Phi$ always facilitate us to consider a typical link, whose receiver is at the origin and the corresponding source node is at $z$ as shown in Fig. 1. 


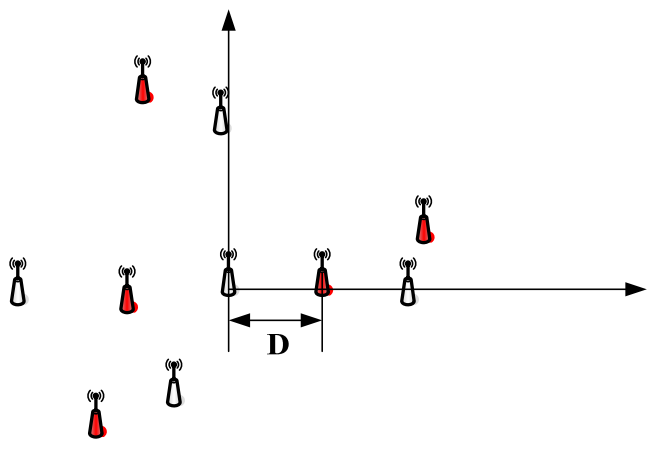

Figure 1. An ad hoc network

The channel contains both large scale path loss and small scales Rayleigh fading. Thus the signal received at the typical receiver can be formulated as

$$
\mathbf{y}=\mathbf{h}_{z} \sqrt{P \ell(\|z\|)} s_{z}+\sum_{x \in \Phi\{z\}} \mathbf{h}_{x} \sqrt{P \ell(\|x\|)} s_{x}+\eta
$$

where $\mathbf{h}_{z}$ is the channel vector of the typical link and equals $\left[\begin{array}{llll}h_{z, 1} & h_{z, 2} & \cdots & h_{z, N}\end{array}\right]^{T}$, where $h_{z, 1} \sim C N(0,1)$, $\ell(\|z\|)=\|z\|^{-\alpha},(\alpha>2)$ represents the path loss effect and $\alpha$ is the path loss exponent [1].

Here the channel fading are assumed to be timecorrelated. Considering the feedback delay, the relation between the beamforming vector and the channel can be expressed through the Gauss-Markov model, i.e.

$$
\mathbf{h}_{z}=\rho \tilde{\mathbf{h}}_{z}+\sqrt{1-\rho^{2}} \mathbf{w}_{z}
$$

$\tilde{\mathbf{h}}_{z}$ is the beamforming vector, $\mathbf{w}_{z}$ is a vector with entries distributed as $C N(0,1)$ and is independent with $\tilde{\mathbf{h}}_{z}$. In order not to violate the power constrain, the beamforming vector is chosen as

$$
\mathbf{v}_{z}=\frac{\tilde{\mathbf{h}}_{z}}{\left\|\tilde{\mathbf{h}}_{z}\right\|}
$$

Thus the SINR of signal after MRT can be formulated as

$$
\gamma_{z}=\frac{\left|\mathbf{v}_{z}{ }^{H} \mathbf{h}_{z}\right|^{2} P \ell(\|z\|)}{\sum_{x \in \Phi\{z\}}\left|\mathbf{v}_{z}{ }^{H} \mathbf{h}_{x}\right|^{2} P \ell(\|x\|)+\sigma^{2}}
$$

where $H$ is the conjugate transpose operation, $\sigma^{2}$ is the power of the thermal noise and is neglected hereafter ${ }^{1}$. Based on (4), the outage probability will be derived next.

\section{OUTAGE ANALYSIS FOR MRT}

Outage probability, which is widely adopted performance parameter, is used to evaluate the performance of MRT with imperfect CSI. Specifically, outage probability is defined as

$$
\mathrm{P}_{\text {out }}=\mathrm{P}\left(\gamma_{z} \leq T\right)=\mathrm{P}\left(\frac{\left|\mathbf{v}_{z}{ }^{H} \mathbf{h}_{z}\right|^{2} \ell(\|z\|)}{\sum_{x \in \Phi\{z\}}\left|\mathbf{v}_{z}{ }^{H} \mathbf{h}_{x}\right|^{2} \ell(\|x\|)} \leq T\right)
$$

Where $T$ is the threshold for correct reception and is determined by the coding and the modulation schemes. From (5), the Cumulative distribution function (CDF) of $g_{z}=\left|\mathbf{v}_{z}{ }^{H} \mathbf{h}_{z}\right|^{2}$ and $g_{x}=\left|\mathbf{v}_{z}{ }^{H} \mathbf{h}_{x}\right|^{2}$ are needed. Since $\mathbf{v}_{z}$ is independent with $\mathbf{h}_{x}, g_{x} \sim \exp (1)$. The CDF of $g_{z}$ can be derived as shown in the following Lemma.

Lemma 1. The CDF of $g_{z}$ can be formulated as

$$
\mathrm{P}\left(g_{z} \leq x\right)=\sum_{i=0}^{N-1}\left(\begin{array}{c}
N-1(6) \\
i(7)
\end{array}\right)\left(1-\rho^{2}\right)^{N-i-1} \rho^{2 i} \frac{\gamma(i+1, x)}{\Gamma(i+1)}
$$

Proof. See Appendix A.

Remark1 : when $\rho=0$, namely, the estimated channel is totally ineffective, $\mathrm{P}\left(g_{z} \leq x\right)$ degenerate to $\gamma(1, x)=1-e^{-x}$. While $\rho=1$, i.e. the channel is estimated perfectly, $\mathbf{P}\left(g_{z} \leq x\right)$ equals $\frac{\gamma(N, x)}{\Gamma(N)}$.

Remark 2 : In order to see how the imperfect CSI affects the performance of MRT, a parameter $\kappa$ is defined as the ratio of the outage probability of single antenna case to the outage probability of the MRC with imperfect CSI, i.e.,

$$
\kappa=\frac{\gamma(1, x)}{\sum_{i=0}^{N-1}\left(\begin{array}{c}
N-1(9) \\
i(10)
\end{array}\right)\left(1-\rho^{2}\right)^{N-1-i} \rho^{2 i} \frac{\gamma(i+1, x)}{\Gamma(i+1)}} .
$$

${ }^{1}$ When the network is interference dominant, the power of the thermal noise is small compared to the interference power and is thus negligible. Furthermore, the derivation can directly follows when the thermal noise is incorporated without significant difference.

From the discussion in Appendix B, the maximum performance gain of MRT with imperfect CSI over single antenna case is

$$
\kappa=\left(1-\rho^{2}\right)^{-N+1}
$$

Based on (8), it obvious $\kappa$ will decrease with $\rho$ decreasing, that is, channel estimation error will degrade the performance of MRT. Meanwhile, a larger $N$ will result in a larger $\kappa$. 
Remark3 : From (6), we can also get some insight about the noise dominant networks. For a noise dominant network, the outage probability equals

$$
\mathrm{P}_{\text {out }}=\mathrm{P}\left(\frac{\left|\mathbf{v}^{H} \mathbf{h}_{z}\right|^{2} P \ell(\|z\|)}{\sigma^{2}} \leq T\right)=\mathrm{P}\left(\left|\mathbf{v}^{H} \mathbf{h}_{z}\right|^{2} \leq \frac{T}{\bar{\gamma}}\right)
$$

where $\bar{\gamma}$ is the average received SNR and equals $\frac{P \ell(\|z\|)}{\sigma^{2}}$. With (6), $\mathrm{P}_{\text {out }}$ can be formulated as

$$
\mathrm{P}_{\text {out }}=\sum_{i=0}^{N-1}\left(\begin{array}{c}
N-1(14) \\
i(15)
\end{array}\right)\left(1-\rho^{2}\right)^{N-i-1} \rho^{2} \frac{\gamma\left(i+1, \frac{T}{\gamma}\right)}{\Gamma(i+1)}
$$

From the derivation in Appendix C, when $\bar{\gamma} \rightarrow \infty$, $P_{\text {out }} \sim O\left(\frac{1}{y}\right)$, where $f(z) \sim O \quad(Z)$ means $\lim _{z \rightarrow 0} \frac{f(z)}{z}$ is bounded. This result indicates $\lim _{z \rightarrow 0} \frac{-\log P_{\text {out }}}{\log \bar{\gamma}}=1$, i.e. only diversity of one can be achieved and this can be demonstrate by Fig. 2. Thus channel estimation error will incur certain diversity loss.

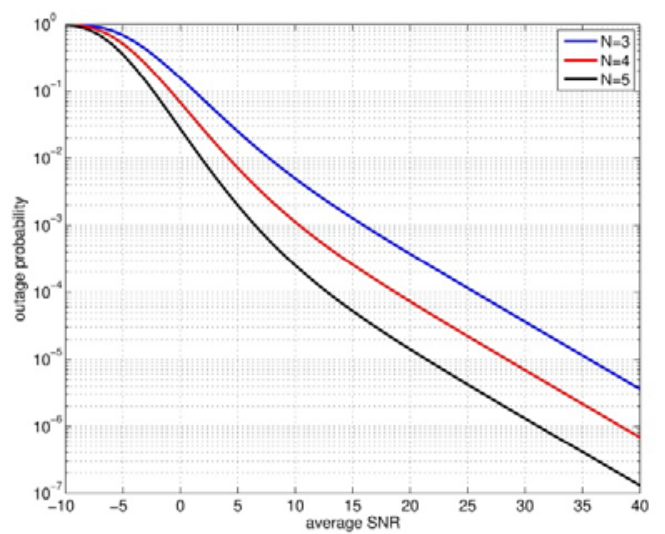

Figure 2. The outage probability v.s. SNR. .

From (5) and (6), $\mathrm{P}_{\text {out }}$ can be reformulated as

$$
\begin{aligned}
\mathrm{P}_{\text {out }} & =\mathrm{E}\left[\mathrm{P}\left(g_{z} \leq T I D^{\alpha}\right)\right] \\
& =\sum_{i=0}^{N-1}\left(\begin{array}{c}
N-1(17) \\
i(18)
\end{array}\right)\left(1-\rho^{2}\right)^{N-1-i} \\
& \rho^{2 i} \mathrm{E}\left[\frac{\gamma(i+1, T I \ell(\|z\|))}{\Gamma(i+1)}\right]
\end{aligned}
$$

where $I=\sum_{x \in \Phi\{z\}} g_{x} \ell(\|x\|)$. Following the approach in [4], $\mathrm{P}_{\text {out }}$ can be derived in closed forms as shown in the following Lemma.

Lemma 2. The outage probability with imperfect CSI equals

$$
\begin{aligned}
& \mathrm{P}_{\text {out }}=1-e^{-\pi \lambda \mathrm{B}\left(1-\frac{2}{\alpha}, 1+\frac{2}{\alpha}\right) T^{\frac{2}{\alpha}} D^{2}} \sum_{i=0}^{N-1}\left(\begin{array}{c}
N-1(20) \\
i(21)
\end{array}\right) \\
& \left(1-\rho^{2}\right)^{N-1-i} \rho^{2 i}\left(\sum_{k=0}^{i} \frac{1}{k !} K_{k}\right)
\end{aligned}
$$

Where $K_{0}=1, K_{1}=\pi \lambda \frac{2}{\alpha} \mathrm{B}\left(1-\frac{2}{\alpha}, 1+\frac{2}{\alpha}\right) T^{\frac{2}{\alpha}} D^{2}$ and for $k>1$,

$$
\begin{aligned}
& K_{k}=\sum_{m=1}^{k}(-1)^{k+m}\left(\pi \lambda \frac{2}{\alpha} \mathrm{B}\left(1-\frac{2}{\alpha}, 1+\frac{2}{\alpha}\right) T^{\frac{2}{\alpha}} D^{2}\right)^{m} \\
& \sum_{\delta_{p} \in M_{m}} \prod_{l_{j} \in \delta_{p}}\left(\frac{2}{\alpha}\left(l_{j}-j\right)-l_{j}+1\right)
\end{aligned}
$$

Proof. See Appendix C.

The outage probability for MRT transmission with CSI of various quality is shown in Fig. 3. According to Fig. 3, the quality of CSI has a non-negligible impact on the performance of MRT. Thus certain attention should be made on the design of the CSI feedback. As shown in Fig. 3 , the outage probability increases with the intensity of source nodes increasing. Moreover, the perfect match between the analytical results and the simulation results verifies our analysis.

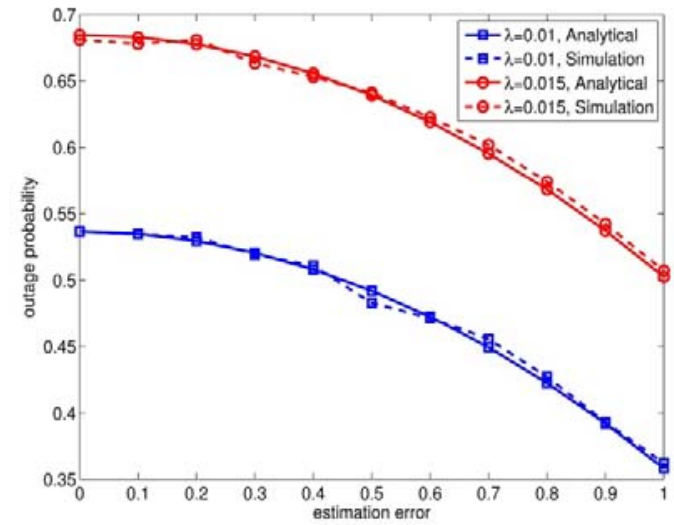

The Outage Probability v.s. Channel Estimation Error .

\section{CONCLUSIONS}

In this paper, the performance of MRT in an ad hoc network was analyzed. By modeling the source nodes as a homogeneous Poisson point process, the outage probability of MRT with imperfect CSI was derived in closed forms. 
From our analysis, the quality of CSI had a great impact on the performance of MRT and the imperfection of CSI would cause certain diversity loss. Thus certain attention should be made on the design of CSI feedback.

\section{A. Appendix A}

Proof of Lemma 1

From the definition of $g_{z}$, we have

$$
\begin{aligned}
g_{z} & =\left|\mathbf{V}_{z}{ }^{H} \mathbf{h}_{z}\right|^{2}=\left|\frac{\tilde{\mathbf{h}}_{z}{ }^{H} \mathbf{h}_{z}}{\left\|\tilde{\mathbf{h}}_{z}\right\|}\right|^{2} \\
& =\left|\rho\left\|\tilde{\mathbf{h}}_{z}\right\|+\sqrt{1-\rho^{2}} \frac{\tilde{\mathbf{h}}_{z}{ }^{H} \mathbf{W}_{z}}{\left\|\tilde{\mathbf{h}}_{z}\right\|}\right|^{2}
\end{aligned}
$$

where $\quad\left\|\tilde{\mathbf{h}}_{z}\right\|$ equals $\sqrt{\sum_{i=1}^{N}\left|\tilde{h}_{i}\right|^{2}}$. Since $\sqrt{1-\rho^{2}} \frac{\tilde{\mathbf{h}}_{z}{ }^{H} \mathbf{w}_{z}}{\left\|\tilde{\mathbf{h}}_{z}\right\|} \sim C N\left(0,1-\rho^{2}\right), \quad$ clearly $g_{z}=|X|^{2}$ given $\left\|\tilde{\mathbf{h}}_{z}\right\|$, where $X \sim C N\left(\rho\left\|\tilde{\mathbf{h}}_{z}\right\|, 1-\rho^{2}\right)$. From [7],

$$
f_{g_{z}\left\|\tilde{\mathbf{h}}_{z}\right\|}(x)=\frac{1}{1-\rho^{2}} e^{-\frac{\rho^{2} \|\left.\tilde{\mathbf{h}}_{z}\right|^{2}+x}{1-\rho^{2}}} I_{0}\left(\frac{2 \sqrt{\rho^{2}\left\|\tilde{\mathbf{h}}_{z}\right\|^{2} x}}{1-\rho^{2}}\right)
$$

where $I_{0}(z)$ is the modified Bessel function of the first kind with order zero defined as $\sum_{k=0}^{\infty} \frac{1}{k ! \Gamma(k+1)}\left(\frac{z}{2}\right)^{2 k}$. The Laplace transform of $g_{z}$ equals

$$
L_{g_{z}\left\|\tilde{\mathbf{h}}_{z}\right\|}(s)=\frac{1}{1+\left(1-\rho^{2}\right) s} e^{-\frac{\rho^{2}\left\|\tilde{h}_{\boldsymbol{h}^{2}}\right\|^{2}}{1+\left(1-\rho^{2}\right) s}}
$$

Since $t=\left\|\tilde{\mathbf{h}}_{z}\right\|^{2} \sqcap \operatorname{Erlang}(N, 1)$, then

$$
\begin{aligned}
L_{g_{z}}(s) & =\frac{\int_{0}^{\infty} L_{g_{z} \mid t}(s) t^{N-1} e^{-t} d t}{(N-1) !} \\
& =\frac{1}{(N-1) !} \frac{\int_{0}^{\infty} e^{-\frac{1+s}{1+\left(1-\rho^{2}\right)} t} t^{N-1} d t}{1+\left(1-\rho^{2}\right) s} \\
& =\frac{\left(1+\left(1-\rho^{2}\right) s\right)^{N-1}}{(1+s)^{N}}
\end{aligned}
$$

Thus the probability density function (PDF) of $g_{z}$ can be derived from $L_{g_{z}}(s)$ through inverse Laplace transform, i.e.,

$$
f_{g_{z}}(x)=\int_{\gamma-i \infty}^{\gamma+i \infty} L_{g_{z}}(s) e^{s x} d s
$$

From (17), $L_{g_{z}}(s)$ has a pole of order $N$ at $s=-1$. According to residue theorem, we have

$$
\begin{aligned}
f_{g_{z}}(x) & =\frac{1}{(N-1) !} \lim _{s \rightarrow-1} \frac{d^{N-1}}{d s^{N-1}}(s+1)^{N} L_{g_{z}}(s) e^{s x} \\
& =\frac{1}{(N-1) !} \lim _{s \rightarrow-1} \frac{d^{N-1}}{d s^{N-1}}\left(1+\left(1-\rho^{2(m-1)}\right) s\right)^{N-1} e^{s x}
\end{aligned}
$$

Based on general Leibniz's rule, we have

$$
\begin{aligned}
\frac{d^{N-1}}{d s^{N-1}}\left(1+\left(1-\rho^{2}\right) s\right)^{N-1} e^{s x}= & \sum_{i=0}^{N-1}\left\{\left(\begin{array}{c}
N-1(30) \\
i(31)
\end{array}\right) x^{i} e^{s x}\right. \\
& \frac{\Gamma(N)}{\Gamma(i+1)} \times\left(1-\rho^{2}\right)^{N-i-1} \\
& \left.\left(1+\left(1-\rho^{2}\right) s\right)^{i}\right\} .
\end{aligned}
$$

Substituting (20)into (19), the PDF of $g_{z}$ can be derived as

$$
f_{g_{z}}(x)=\sum_{i=0}^{N-1}\left(\begin{array}{c}
N-1(33) \\
i(34)
\end{array}\right) \frac{\left(1-\rho^{2}\right)^{N-i-1} \rho^{2 i}}{\Gamma(i+1)} x^{i} e^{-x} \text {. }
$$

Thus the CDF of $g_{z}$ follows

$$
\begin{aligned}
\mathrm{P}\left(g_{z} \leq x\right) & =\sum_{i=0}^{N-1}\left(\begin{array}{c}
N-1(36) \\
i(37)
\end{array}\right) \frac{\left(1-\rho^{2}\right)^{N-i-1} \rho^{2 i}}{\Gamma(i+1)} \int_{0}^{x} t^{i} e^{-t} d t \\
& =\sum_{i=0}^{N-1}\left(\begin{array}{c}
N-1(38) \\
i(39)
\end{array}\right) \frac{\left(1-\rho^{2}\right)^{N-i-1} \rho^{2 i}}{\Gamma(i+1)} \gamma(i+1, x) .
\end{aligned}
$$

\section{B. Appendix $B$}

Derivation of Maximum of $\kappa$

From (7), in order to get the maximum of $\kappa$, we can just derive the minimum of $\frac{1}{\kappa}$, namely 


$$
\tau=\sum_{i=0}^{N-1}\left(\begin{array}{c}
N-1(41) \\
i(42)
\end{array}\right) \frac{\left(1-\rho^{2}\right)^{N-1-i} \rho^{2 i}}{\Gamma(i+1)} \frac{\gamma(i+1, x)}{\gamma(1, x)} .
$$

Taking the first order derivative of $\frac{\gamma(i+1, x)}{\gamma(1, x)}$, it follows

$$
\begin{aligned}
\frac{d}{d x} \frac{\gamma(i+1, x)}{\gamma(1, x)} & =\frac{x^{i} e^{-x} \gamma(1, x)-e^{-x} \gamma(i+1, x)}{\gamma(1, x)^{2}} \\
& \stackrel{(a)}{=} \frac{e^{-x} \int_{0}^{x} x^{i} e^{-t} d t-e^{-x} \int_{0}^{x} t^{i} e^{-t} d t}{\gamma(1, x)^{2}}>0,
\end{aligned}
$$

where $(a)$ follows from the definition of incomplete gamma function. Thus $\frac{\gamma(i+1, x)}{\gamma(1, x)}$ is an increasing function, and $\tau$ achieves its minimum when $x \rightarrow 0$, i.e.,

$$
\begin{aligned}
\tau_{\min } & =\lim _{x \rightarrow 0} \tau \\
& =\sum_{i=0}^{N-1}\left(\begin{array}{c}
N-1(45) \\
i(46)
\end{array}\right) \frac{\left(1-\rho^{2}\right)^{N-1-i} \rho^{2 i}}{\Gamma(i+1)} \lim _{x \rightarrow 0} \frac{\gamma(i+1, x)}{\gamma(1, x)} .
\end{aligned}
$$

According

$$
\text { to }
$$

$$
\gamma(i+1, x)=\frac{1}{i+1} x^{i+1} e^{-x} M(1, i+2, x), M(a, b, z) \text { is }
$$

the Kummer function defined as $\sum_{s=0}^{\infty} \frac{(a)_{s}}{(b)_{s} s !} z^{s}$. Then we have

$$
\frac{\gamma(i+1, x)}{\gamma(1, x)}=\frac{1}{i+1} x^{i} \frac{M(1, i+2, x)}{M(1,2, x)} \text {. }
$$

Since $M(a, b, z)=1+O(z)^{1}$, when $z \rightarrow 0$, we can get

$$
\lim _{x \rightarrow 0} \frac{M(1, i+2, x)}{M(1,2, x)}=\frac{\lim _{x \rightarrow 0} M(1, i+2, x)}{\lim _{x \rightarrow 0} M(1,2, x)}=1 .
$$

Then it can be concluded $\frac{\gamma(i+1, x)}{\gamma(1, x)}=o(x)$, where $f(z)=o(z)$ means $\left|\frac{f(z)}{z}\right| \rightarrow 0$ when $x \rightarrow 0$. Thus $\tau_{\text {min }}$ equals $\left(1-\rho^{2}\right)^{N-1}$, and the maximum of $\kappa$ equals $\left(1-\rho^{2}\right)^{-N+1}$.

\section{Appendix C}

Derivation of Diversity order
According to [8], we have

$$
\left(1-e^{-\frac{T}{\Gamma(i+2) \frac{1}{i+1}}}\right)^{i+1} \leq \frac{\gamma\left(i+1, \frac{T}{\bar{\gamma}}\right)}{\Gamma(i+1)} \leq\left(1-e^{-\frac{T}{a r \gamma}}\right)^{i+1}
$$

Thus $\mathrm{P}_{\text {out }}$ in (9) can be upper bounded as

$$
\mathrm{P}_{\text {out }} \leq\left(1-e^{-\frac{T}{\bar{P}}}\right)\left(1-\rho^{2} e^{-\frac{T}{\bar{P}}}\right)^{N-1} .
$$

${ }^{2} f(z)=O(z)$ means $\left|\frac{f(z)}{z}\right|$ is bounded when $z \rightarrow 0$.

Next, a lower bound on $\mathrm{P}_{\text {out }}$ will be derived. Taking $\nabla$ as the ration between $\Gamma(i+2)^{i+2}$ and $\Gamma(i+3)^{i+1}$, i.e.,

$$
\nabla=\frac{\Gamma(i+2)^{i+2}}{\Gamma(i+3)^{i+1}}=\frac{(i+1) !}{(i+2)^{i+1}} .
$$

From Arithmetic-Geometric Average Inequality, we have

$$
\nabla \leq \frac{\left(\frac{i+2}{2}\right)^{i+1}}{(i+2)^{i+1}}<1
$$

Thus we can conclude $\Gamma(i+2)^{i+2}<\Gamma(i+3)^{i+1}$, namely $\Gamma(i+2)^{\frac{1}{i+1}}<\Gamma(i+3)^{\frac{1}{i+2}}$. Then $\mathrm{P}_{\text {out }}$ can be lower bounded as

$$
\begin{aligned}
\mathrm{P}_{\text {out }} & >\sum_{i=0}^{N-1}\left(\begin{array}{c}
N-1(54) \\
i(55)
\end{array}\right)\left(1-\rho^{2}\right)^{N-i-1} \rho^{2}\left(1-e^{-\frac{T}{\Gamma(N+1) \frac{1}{N+1 Y}}}\right)^{i+1} \\
& =\left(1-e^{-\frac{T}{\Gamma(N+1) \frac{1}{N+1 Y}}}\right)\left(1-\rho^{2} e^{-\frac{T}{\Gamma(N+1) \frac{1}{N+1 Y}}}\right)^{N-1} .
\end{aligned}
$$

When $\bar{\gamma} \rightarrow \infty$, the upper bound of follows

$$
\mathrm{P}_{\text {out }} \stackrel{(a)}{\sim} \frac{T}{\bar{\gamma}}\left(1-\rho^{2}+\rho^{2} \frac{T}{\bar{\gamma}}\right)^{N-1} \sim\left(1-\rho^{2}\right)^{N-1} \frac{T}{\bar{\gamma}},
$$

where $(a)$ follows from $e^{-x} \sim x$. Similarly, the lower bound can be followed as

$$
\begin{aligned}
& \mathrm{P}_{\text {out }} \beth \frac{T}{\Gamma(N+1)^{\frac{1}{N+1}} \bar{\gamma}}\left(1-\rho^{2}+\rho^{2} \frac{T}{\Gamma(N+1)^{\frac{1}{N+1}} \bar{\gamma}}\right)^{N+1} \\
& \beth \frac{T}{\Gamma \operatorname{left}(N+1}{ }^{\frac{1}{N+1}} \bar{\gamma}\left(1-\rho^{2}\right)^{N-1} .
\end{aligned}
$$


From (33) and (34), it is obvious when $\bar{\gamma} \rightarrow \infty, \mathrm{P}_{\text {out }}$ decreases as $O\left(\frac{1}{\bar{\gamma}}\right)$.

\section{Appendix D}

\section{Proof of Lemma 2} have

From the property of incomplete gamma function, we

$$
\xi=\frac{\gamma\left(i+1, T I D^{\alpha}\right)}{\Gamma(i+1)}=1-e^{-T I D^{\alpha}} \sum_{k=0}^{i} \frac{\left(T I D^{\alpha}\right)^{k}}{k !} .
$$

Where $D=\|z\|$. Following [4], (59) can be reformulated as

$$
\xi=1-\left.\sum_{k=0}^{i} \frac{\left(-T D^{\alpha}\right)^{k}}{k !} \frac{d^{k}}{d s^{k}} e^{-s I}\right|_{s=T D^{\alpha}}
$$

Thus (11) can be formulated as

$$
\begin{aligned}
\mathrm{P}_{\text {out }}= & 1-\sum_{i=0}^{N-1}\left\{\left(\begin{array}{c}
N-1(61) \\
i(62)
\end{array}\right)\left(1-\rho^{2}\right)^{N-1-i} \rho^{2 i}\right. \\
& \times\left(\sum_{k=0}^{i} \frac{(-1)^{k}\left(T D^{\alpha}\right)^{k}}{k !} \frac{d^{k}}{d s^{k}} \mathrm{E} \leq f t\left[e^{-s I}\right]_{s=T D^{\alpha}}\right\}
\end{aligned}
$$

Based on Slivnyak theorem and the laplace transform of the homogeneous poisson point process, it follows

$$
\mathrm{E}\left[e^{-s I}\right]=e^{-\pi \lambda \mathrm{B}\left(1-\frac{2}{\alpha}, 1+\frac{2}{\alpha}\right) s^{\frac{2}{\alpha}}} .
$$

The derivative in (38) can be formulated as

$$
\begin{aligned}
\frac{d^{k}}{d s^{k}} e^{-\pi \lambda \mathrm{B}\left(1-\frac{2}{\alpha}, 1+\frac{2}{\alpha}\right) s^{\frac{2}{\alpha}}}= & e^{-\pi \lambda \mathrm{B}\left(1-\frac{2}{\alpha}, 1+\frac{2}{\alpha}\right) s^{\frac{2}{\alpha}}} \sum \text { limits }_{m=1}^{k} \\
& \left\{\left(-\pi \lambda \frac{2}{\alpha} \mathrm{B}\left(1-\frac{2}{\alpha}, 1+\frac{2}{\alpha}\right)\right)^{m}\right. \\
& \times s^{m \frac{2}{\alpha}-k} \sum_{\delta_{p} \in M_{m}} \prod t s_{l_{j} \in \delta_{p}} \\
& \left.\left(\frac{2}{\alpha}\left(l_{j}-j\right)-l_{j}+1\right)\right\}
\end{aligned}
$$

where $M_{m}$ is the set formed by all the subsets of the natural number set $\{2, \cdots, k\}$ of cardinality $k-m$ and the subscript $j$ in $l_{j}$ means $l_{j}$ is the $j$ th time that the derivative is taken on the polynomial term. With (37) and (39), $\mathrm{P}_{\text {out }}$ can be derived as in (11).

\section{REFERENCES}

[1] A. Goldsmith, Wireless Communications, Cambridge: Cambridge press, 2005.

[2] E. N. Onggosanusi, A. Gatherer, A. G. Dabak and S. Hosur, "Performance analysis of closed-loop transmit diversity in the presence of feedback delay," IEEE Trans. Commun., vol. 49, pp. 1618-1630, Sep. 2001

[3] N.-S. Kim and Y. H. Lee, "Effect of channel estimation errors and feedback delay on the performance of closed-loop transmit diversity system," in Proc. IEEE SPAWC, Jun. 2003.

[4] A. M. Hunter, J. G. Andrews and S. Weber, "Transmission capacity of ad hoc networks with spatial diversity," IEEE Trans. Wireless Commun., vol. 7, no. 12, pp. 5058-5071, Dec. 2008.

[5] F. Baccelli, B. Błaszczyszyn, Foundations and Trends in Networking: Stochastic Geometry and Wireless Networks (volume 1), Boston, MA: Now Publishers, 2009.

[6] F. Baccelli, B. Błaszczyszyn, Foundations and Trends in Networking: Stochastic Geometry and Wireless Networks (volume 2), Boston, MA: Now Publishers, 2009.

[7] D. Stoyan, W. Kendall and J. Mecke, Stochastic Geometry and its Applications, Chichester: WILEY, 1995.

[8] F. W. J. Olver, D. W. Lozier, R. F. Boisvert, and C. W. Clark, NIST Handbook of Mathematical Functions, New York, NY: Cambridge University Press, 2010.

[9] .S. Gradshteyn and I.M. Ryzhik, Tables of Integrals, Series, and Products, San Diego: Academic press, 2007 